\title{
Relationship Between the Closed Kinetic Chain Upper Extremity Stability Test and Strength of Serratus Anterior and Triceps Brachii Muscles
}

\author{
Young-soo Weon ${ }^{1,2}$, BPT, PT, Sun-hee Ahn ${ }^{2,3}, \mathrm{PhD}, \mathrm{PT}$, Jun-hee Kim²,3, PhD, PT, Gyeong-tae Gwak ${ }^{1,2}, \mathrm{BPT}, \mathrm{PT}$, \\ Oh-yun Kwon ${ }^{2,3}$, PhD, PT \\ ${ }^{1}$ Department of Physical Therapy, The Graduate School, Yonsei University, ${ }^{2}$ Kinetic Ergocise Based on Movement Analysis Laboratory, \\ ${ }^{3}$ Department of Physical Therapy, College of Health Science, Yonsei University, Wonju, Korea
}

\author{
Article Info \\ Received July 5, 2021 \\ Revised July 13, 2021 \\ Accepted July 16, 2021 \\ Corresponding Author \\ Oh-yun Kwon \\ E-mail: kwonoy@yonsei.ac.kr \\ https://orcid.org/0000-0002-9699-768X
}

\section{Key Words}

Muscle strength

Physical functional performance

Shoulder joint
Background: The CKCUES test evaluates the functional performance of the shoulder joint. The CKCUES test scores CKC exercises of the upper limbs to examine shoulder stability. Although the CKCUES test provides quantitative data on functional ability and performance, no study has determined the relationship between CKCUES scores and SA and TB muscle strength.

Objects: The objective of this study is to determine the relationship between the CKCUES test scores and the strength of the SA and TB muscles in the CKCUES and unilateral CKCUES tests. Methods: Sixty-six healthy male volunteers participated in the study. A Smart KEMA strength sensor measured SA and TB muscle strength. Two parallel lines on the floor indicated the initial hand placement to start CKCUES tests. For 15 seconds, the subject raises one hand and reaches over to touch the supporting hand, then returns to the starting position.

Results: The correlation between the CKCUES test scores and the strength of the SA was strong $(r=0.650, p<0.001)$, and the TB was moderate $(r=0.438, p<0.001)$. The correlation between the unilateral CKCUES test and the strength of the SA of the supporting side was strong $(r=0.605, p<0.001)$, and swing side was strong $(r=0.681, p<0.001)$. The correlation between the unilateral CKCUES test and the strength of the TB of the supporting side was moderate $(r=0.409, p<0.001)$, and swing side was moderate $(r=0.482, p<0.001)$.

Conclusion: Our study showed that the CKCUES test had a strong association with isometric strength of SA and moderate association with that of TB. These findings suggest that the CKCUES test can evaluate the function of the SA. Moreover, the unilateral CKCUES test can evaluate unilateral shoulder function.

\section{INTRODUCTION}

The shoulder joint is used in daily physical activities, and people can experience various complex joint problems. Shoulder pain and functional disorders are associated with scapular positional impairments and abnormal movements caused by scapular instability [1]. Unstable scapula may lead to inefficiency in the rotator cuff muscles to control the humeral head in the glenoid fossa during overhead elevation. This can contribute to a rupture of the rotator cuff and symptoms of impingement syndrome [2-4].

The serratus anterior (SA) contributes to stability during scapula motions like upward rotation, posterior tilt, and pro- traction [5], and its normal function is essential in maintaining scapulohumeral rhythm during arm elevation [6]. Inman et al. [7] shows that the SA is the key muscle stabilizing the scapula. Insufficient SA strength is linked to shoulder disorders like winging [8], which increases likelihood of shoulder impingement because scapular upward rotation and protraction are deficient during shoulder abduction [9]. Therefore, muscle strength of scapular stabilizers are essential for rehabilitation and prevention of shoulder disorders and dysfunctions associated with scapular instability.

The clinic, sports, and fitness fields use several tests to determine scapular stability and functionality. These include the lateral scapular slide test [10], scapular load test [11], eccentric 
hold test [11], dynamic movement testing, like the one-arm hop test [12], upper quarter Y balance test [13], and closed kinetic chain upper extremity stability test (CKCUES test) [14]. Increased encouragement of functional tests stem from their simplicity, low-cost, and ability to provide vital information on functional performance [15]. Dynamic movement testing, like the one-arm hop test and upper quarter $\mathrm{Y}$ balance test, is gaining popularity in muscular screening to help identify increased injury risk $[13,16,17]$. The CKCUES test is an interesting option for evaluating the stability and functional performance of the shoulder joint [18].

The CKCUES test evaluates the functional performance of the shoulder joint [14]. The CKCUES test scores closed kinetic chain exercises of the upper limbs to examine shoulder stability [14]. It is a simple, low-cost test easily applied in rehabilitation or sports contexts [15]. The subject counts the number of times they touch their other hand for 15 seconds while pushing up. Several studies with a test-retest design found satisfactory reliability levels of CKCUES test scores [14,19]. In addition, the test shows good correlation with grip strength and the peak torque of internal/external shoulder rotation [20].

Although the CKCUES test provides quantitative data on functional ability and performance, no study has determined the relationship between CKCUES scores and SA and triceps brachii (TB) muscle strength. Both provide stability while maintaining a push-up position. In addition, CKCUES starts from the push-up position while touching the hand alternatively. Each side can interdependently affect CKCUES scores. Since the test starts from the push-up position with both hands 36-inches apart, SA and TB strength is essential for supporting body weight. The right side of the scapular and elbow stability can influence the left arm performance for raising and reaching over to touch the right hand. If an individual has weak scapula and/or elbow stabilizers on the right side and normal stability on the left, the CKCUES test score will be low. Alternatively, to raise the hand requires a strong concentric contraction strength of the scapular protractor. The traditional CKCUES test cannot distinguish between right and leftside dysfunctions. Therefore, we assumed that the unilateral CKCUES test (one hand supports and the opposite raises and touches the supporting hand and then returns without alternation) can identify the weaker stabilizing side or the weaker swing side separately. Study of the relationship between the CKCUES test and strength of SA and TB muscles in CKCUES and unilateral CKCUES tests would be useful to evaluate and treat shoulder dysfunction related to scapular instability.

Therefore, the first objective of this study is to determine the relationship between the CKCUES test scores and the strength of the SA and TB muscles. And, second objective is to determine the relationship between the unilateral CKCUES test scores and the strength of the SA and TB muscles.

\section{MATERIALS AND METHODS}

\section{Subjects}

Sixty-six healthy male volunteers (age: $23.52 \pm 3.85$ years, body weight: $77.36 \pm 5.03 \mathrm{~kg}$, height: $175.42 \pm 9.69 \mathrm{~cm}$ ) participated in the study (Table 1). All participants were injury free, especially in the shoulder, elbow, and wrist joints. The exclusion criteria were (1) history of tears in any muscles and tendons of the shoulder complex, (2) history of subluxation or osteoarthrosis in the glenohumeral or acromioclavicular joints, (3) rheumatoid, neurological, or degenerative disease, (4) positive results on Adison and Allen tests, and (5) could not assume the push-up position due to back and extremity pain. The Yonsei University Mirae Campus Human Studies Committee (approval number: 1041849-202002-BM-019-02) approved the study procedure, and all participants provided written informed consent.

\section{CKCUES Test and Unilateral CKCUES Test}

To start, subjects assumed a push-up position, hands 36-inches apart, and weight-bearing upper extremities positioned perpendicular to the floor and over the hands. Two parallel lines on the floor indicated the initial hand placement. For 15 seconds, the subject raises one hand and reaches over to touch the supporting hand, then returns to the starting position. To determine the relationship between the strength of the swing and supporting sides, we performed unilateral CKCUES tests (Figure 1).

The unilateral CKCUES test has one hand supporting body

Table 1. Characteristics of the subjects

\begin{tabular}{lr}
\hline Characteristics & Male $(\mathrm{n}=66)$ \\
\hline Age $(\mathrm{y})$ & $23.52 \pm 3.85$ \\
Body height $(\mathrm{cm})$ & $175.42 \pm 9.69$ \\
Body weight $(\mathrm{kg})$ & $77.36 \pm 5.03$ \\
\hline
\end{tabular}

Values are presented as mean \pm standard deviation. 


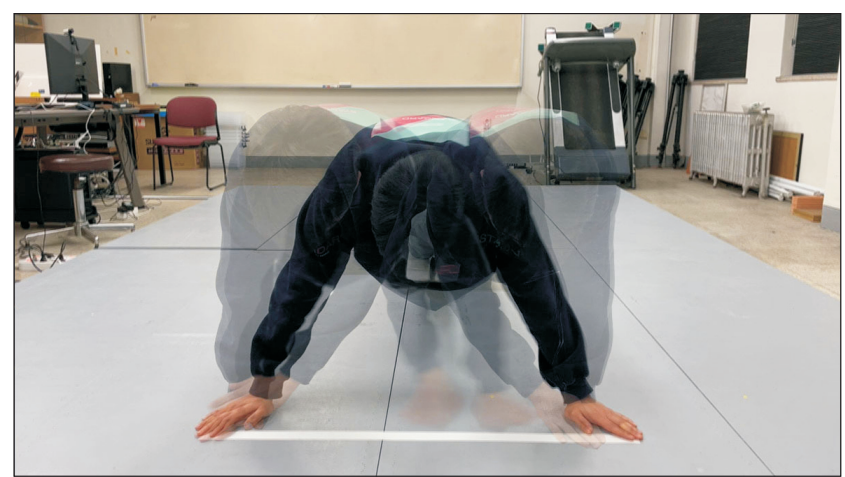

Figure 1. Closed kinetic chain upper extremity stability test.

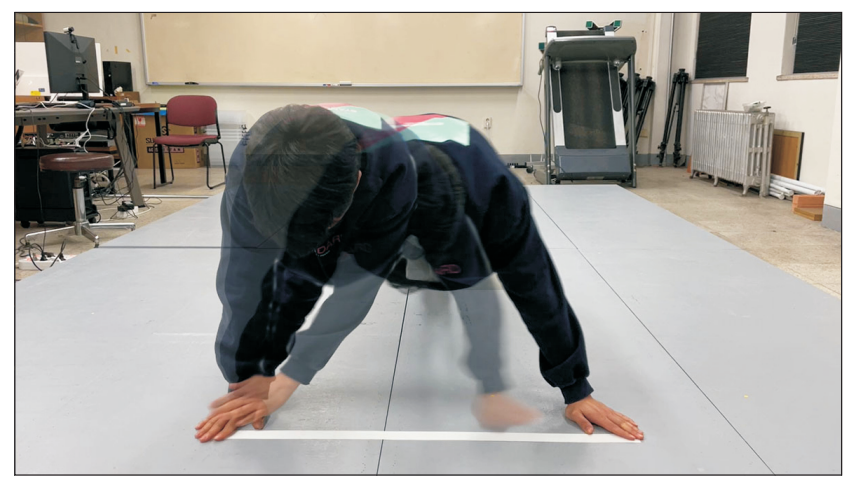

Figure 2. Unilateral closed kinetic chain upper extremity stability test.

weight throughout the test. The other hand raises and touches the supporting hand, then returns to the starting position repeatedly without changing the swing or alternating hands. The test was performed on the right and left sides separately, based on the supporting side. The right unilateral CKCUES test refers to the right-hand supporting side and the swing hand on the left (Figure 2). The principal investigator (PI) demonstrated both the CKCUES and unilateral CKCUES tests for the subjects. After receiving instructions and a demonstration, each subject practiced familiarization with both tests. Then, for data collection, each subject performed two trials for 15 seconds. For the CKCUES test, subjects were asked to touch the supporting hand with the swing hand alternately as quickly as possible for 15 seconds. For the right-side unilateral CKCUES test, subjects were asked to support their body weight with their right hand, raise their left hand, touch the right hand, then return to the starting position repeatedly. For the left-side unilateral CKCUES test, subjects were asked to support their body weight with their left hand, raise their right hand, and touch their left hand. PI counted the number of touches (score). There was a time rest of 45 seconds between trials. A work/rest ratio of 1:3

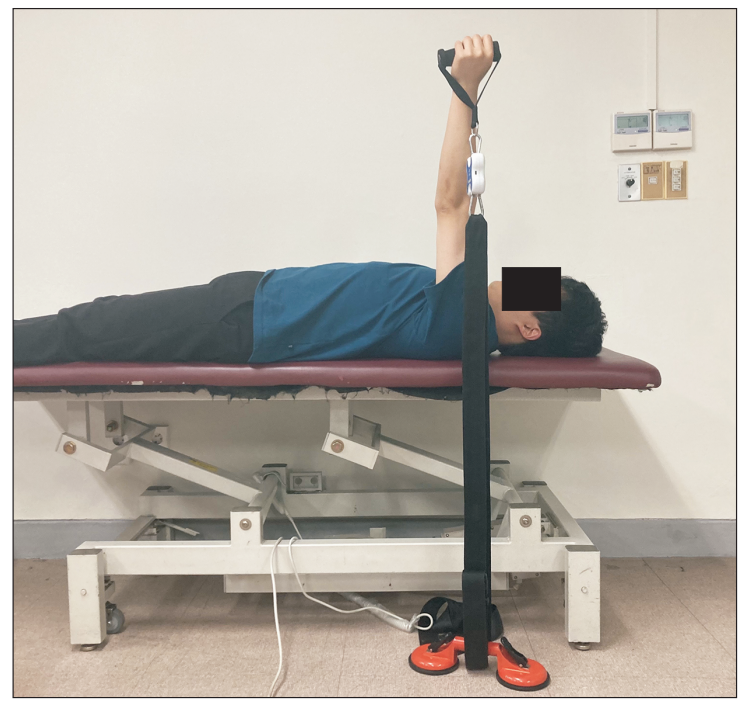

Figure 3. Measurement of strength of the serratus anterior.

can help avoid fatigue effects during a short and relatively high intensity test, like the CKCUES test [19]. We randomly selected the test orders.

\section{Measurement of Strength}

The study used a Smart KEMA strength measurement system (Smart KEMA strength sensor; KOREATECH Inc., Seoul, Korea) to measure the isometric strength of SA and TB in $\mathrm{kg}$. We then calculated the average of the two trials. We used load cell to measure static forces of 0-199.9 kg with an accuracy of $0.1 \mathrm{~kg}$ $\pm 2 \%$. Smart KEMA software recorded the average strength. In previous studies, Smart KEMA shoulder strength measures showed good to high intra-rater reliability (0.85 to 0.90$)$ [21].

Isometric strength measurements of $\mathrm{SA}$ took place in the supine position. Subjects flexed the shoulder joint at $90^{\circ}$ and grasped the strap handle connected to the Smart KEMA strength sensor, perpendicular to the ground. The initial tension of the strap was set to $2 \mathrm{~kg}$. The subject performed scapular protraction with maximum force toward the ceiling. The subject's trunk was fixed with an orthopedic belt to minimize trunk rotation. To minimize contraction of the pectoralis major, we asked the subject not to adduct and internally rotate the shoulder joint during protraction of the scapula (Figure 3).

We measured the isometric strength of TB in the supine position. The Smart KEMA tension strap was placed on the distal forearm, $3 \mathrm{~cm}$ above the wrist joint. Subjects flexed the shoulder and elbow joint at $90^{\circ}$ and supinated the forearm. Before measuring TB strength, the initial tension of the strap was set 
to $2 \mathrm{~kg}$. Subjects performed elbow extension maximally against a strap. Subjects held their tested elbow with the opposite hand to stabilize (Figure 4).

\section{Statistical Analyses}

Statistical analysis was performed using the SPSS for Windows ver. 25.0 software (IBM Co., Armonk, NY, USA). A Shapiro-Wilk test was used to assess whether the scores of the CKCUES and unilateral CKCUES tests and the isometric strength of the SA and TB were normally distributed. The study found that they were, so Pearson's correlation was used to determine the relationship between the test scores and the isometric strength of SA and TB. The strength of association was classified based on those of the British Medical Journal: 0-0.19 very weak, 0.2-0.39 weak, 0.40-0.59 moderated, 0.6-0.79 as strong, and $0.8-1$ very strong [22]. In all analyses, statistical significance was set at $\mathrm{p}<0.05$.

\section{RESULTS}

Table 2 shows the mean and standard deviation of the CKCUES test scores. Table 3 shows the mean and standard devia-

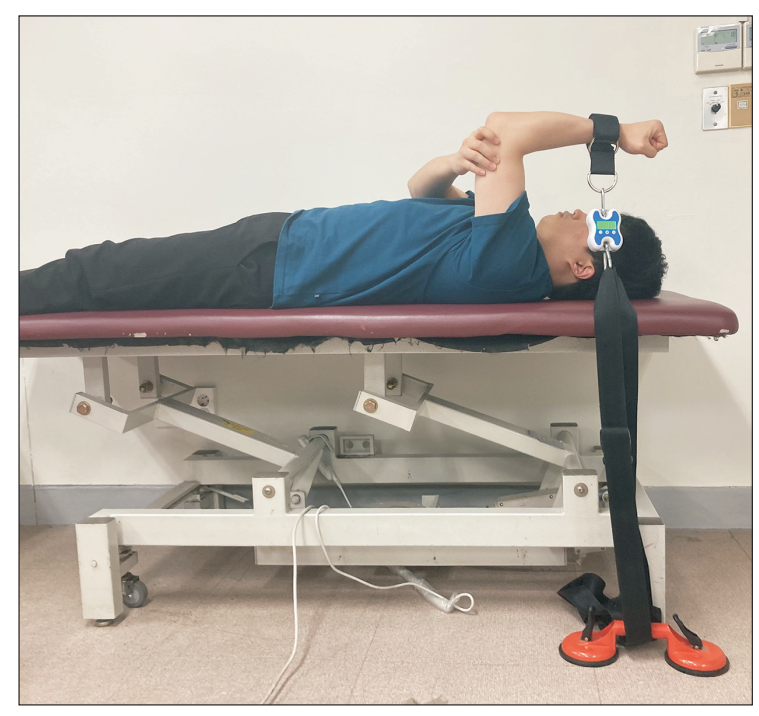

Figure 4. Measurement of strength of the triceps brachii. tion of the strength of SA and TB. The correlation between the CKCUES test scores and the strength of the SA (average of both sides) was strong $(r=0.650, p<0.001)$, and the TB was moderate $(\mathrm{r}=0.438, \mathrm{p}<0.001)$ (Table 4). The correlation between the unilateral CKCUES test and the strength of the SA of the supporting side was strong $(r=0.605, p<0.001)$, and swing side was strong $(r=0.681, p<0.001)$. The correlation between the unilateral CKCUES test and the strength of the TB of the supporting side was moderate $(r=0.409, p<0.001)$, and swing side was moderate $(r=0.482, p<0.001)$ (Table 5).

\section{DISCUSSION}

This study investigated the relationship between CKCUES test scores and isometric strengths of SA and TB. The CKCUES test had a strong association with the isometric strength of SA and moderate association with that of TB. The CKCUES test is widely used as a dynamic shoulder stability function test. In this study, the mean score of the CKCUES test was 30.87. These results are consistent with Tucci et al. [14], who demonstrated a mean CKCUES test score of 27.13 for young healthy males.

The push-up position is widely recommended for recruiting scapular muscles [23], especially in shoulder rehabilitation [24]. Specifically, in many studies the position is used to strengthen the SA [25]. Mosely et al. [26] included the standard push-up plus as a core exercise in their shoulder rehabilitation program based on the high electromyographic activity of several shoulder muscles, including the SA. Park et al. [27] investigated electromyography of SA during various push-up plus exercises. They showed that standard push-up plus is an optimal exercise for subjects with scapular winging, with maximum activation

Table 2. CKCUES test

\begin{tabular}{lc}
\hline \multicolumn{1}{c}{ Variable } & Score \\
\hline CKCUES test (number) & $16.67 \pm 4.52$ \\
Rt side unilateral CKCUES test (number) & $12.30 \pm 3.35$ \\
Lt side unilateral CKCUES test (number) & $12.53 \pm 3.11$ \\
\hline
\end{tabular}

Values are presented as mean \pm standard deviation. CKCUES test, closed kinetic chain upper extremity stability test; Rt, right; Lt, left.

Table 3. Strength of SA and TB

\begin{tabular}{ccrr} 
& Average of both side $(\mathrm{n}=66)$ & Right side $(\mathrm{n}=132)$ & Left side $(\mathrm{n}=132)$ \\
\hline SA strength $(\mathrm{kg})$ & $30.87 \pm 8.61$ & $30.75 \pm 8.41$ & $30.98 \pm 9.22$ \\
TB strength $(\mathrm{kg})$ & $16.48 \pm 4.86$ & $6.61 \pm 5.44$ & $6.61 \pm 5.44$ \\
\hline
\end{tabular}

Values are presented as mean \pm standard deviation. SA, serratus anterior; TB, triceps brachii. 
of the SA and minimal activation of the pectoralis major [27]. This is the first study to investigate the relationship between the CKCUES test and SA strength. The SA is one of the most important muscles for maintaining normal scapular alignment in the shoulder joint. It helps prevent winging or anterior tilting of the scapula [28]. Celik et al. [29] showed that subjects with shoulder impingement syndrome have lower SA strength on the impinged side. Tucci et al. [14] reported that the CKCUES test score was lower in subjects with shoulder impingement syndrome than in those without. These results indicate that the CKCUES test can evaluate the strength of SA and can identify and monitor the risk of shoulder impingement syndrome.

Previous studies have modified the CKCUES test in various ways. Tucci et al. [14] applied the CKCUES test in a kneeling position to evaluate females. Taylor et al. [30] states that having a narrower shoulder width or shorter arm span places athletes at a disadvantage when performing the CKCUES test. For this reason, they modified the CKCUES test to place the hands directly under their shoulders [30]. Degot et al. [31] suggested that a fourth 1 minute set performed after a 15-second recovery following the third set can reflect muscular endurance capacity. However, because all CKCUES test methods evaluate both hands alternately, it was unclear whether the strength of the swing or supporting sides was more significant. In our results, the correlation between the unilateral CKCUES test scores and the strength of the swing side SA and TB was higher than the supporting side $(r=0.681$ vs. $r=0.635)$. The correlation between the unilateral CKCUES test scores and the strength of the swing side SA was higher than between the CKCUES test scores and the average strength of the SA on both sides $(r=$

Table 4. Correlation between the CKCUES test scores and average strength of both sides

\begin{tabular}{ccc} 
Variable & $\begin{array}{c}\text { SA average strength of } \\
\text { both sides }\end{array}$ & $\begin{array}{c}\text { TB average strength of } \\
\text { both sides }\end{array}$ \\
\hline CKCUES test & $0.650^{* *}$ & $0.438^{* *}$ \\
\hline
\end{tabular}

CKCUES test, closed kinetic chain upper extremity stability test; SA, serratus anterior; TB, triceps brachii. ${ }^{* *} p<0.05$.
0.681 vs. $r=0.650)$. This indicates that the SA strength of the swing side is more strongly correlated with the unilateral CKCUES test score than the strength of the supporting side during the CKCUES test. This may be because the swing side requires concentric contraction strength to raise the hand and lift body weight with scapular protraction than stabilizing the scapula on the supporting side. Although SA strength in the swing and supporting sides were similar, the correlation was slightly different between both sides in the unilateral CKUES test $(r=$ 0.681 vs. $r=0.635)$. When the CKCUES test was performed for individuals with similar SA strength in both sides, the unilateral CKCUES test could identify the weaker side.

With the CKCUES test, the isometric strength of TB showed a relatively lower relationship (moderated) than SA. This result is likely because elbow rocking is possible during the CKCUES test. Torres et al. [32] showed no significant difference in muscle activity of TB with or without a stable surface during pushup plus. Lehman et al. [33] showed that due to the mechanical advantage of TB in forearm length, responding to changes in stability during push-ups may be difficult. Therefore, if the strength of TB for the CKCUES test is insufficient, elbow rocking can compensate.

Our study had several limitations. First, our results cannot be generalized because all subjects were male. Further research is necessary to establish the application to women. Second, the core stability and strength of other upper extremity muscles involved in performing the CKCUES test were not measured. Third, since only healthy subjects participated, it is unclear whether the CKCUES test scores are different in subjects with unilateral shoulder disorders. Further research is necessary to determine whether the unilateral CKCUES test scores are different between the normal and impaired sides in subjects with unilateral shoulder disorders.

\section{CONCLUSIONS}

Our study showed that the CKCUES test had a strong association with isometric strength of SA and moderate association

Table 5. Correlation between the unilateral CKCUES test and strength of SA and TB

\begin{tabular}{ccccc}
\hline Variable & $\begin{array}{c}\text { SA strength of } \\
\text { supporting side }\end{array}$ & $\begin{array}{c}\text { SA strength of } \\
\text { swing side }\end{array}$ & $\begin{array}{c}\text { TB strength of } \\
\text { supporting side }\end{array}$ & $\begin{array}{c}\text { TB strength of } \\
\text { swing side }\end{array}$ \\
\hline Unilateral CKCUES test & $0.635^{* *}$ & $0.438^{* *}$ & $0.409^{* *}$ & $0.482^{* *}$ \\
\hline
\end{tabular}

CKCUES test, closed kinetic chain upper extremity stability test; SA, serratus anterior; TB, triceps brachii. ${ }^{* *} p<0.05$. 
with that of TB. These findings suggest that the CKCUES test can evaluate the function of the SA. Moreover, the unilateral CKCUES test can evaluate unilateral shoulder function.

\section{ACKNOWLEDGEMENTS}

This study was supported by the "Brain Korea 21 FOUR Project", the Korean Research Foundation for Department of Physical Therapy in the Graduate School of Yonsei University.

\section{CONFLICTS OF INTEREST}

No potential conflict of interest relevant to this article was reported.

\section{AUTHOR CONTRIBUTIONS}

Conceptualization: YW, OK. Data curation: YW. Formal analysis: YW, SA, JK, GG. Investigation: YW. Methodology: YW, SA, JK, GG, OK. Resources: YW, SA, JK, GG, OK. Supervision: SA, JK, GG, OK. Validation: SA, JK, GG, OK. Visualization: YW. Writing - original draft: YW. Writing - review \& editing: SA, JK, GG, OK.

\section{ORCID}

Young-soo Weon, https://orcid.org/0000-0003-3852-606X

Sun-hee Ahn, https://orcid.org/0000-0001-7660-8598

Jun-hee Kim, https://orcid.org/0000-0002-0498-7207

Gyeong-tae Gwak, https://orcid.org/0000-0003-1812-8910

\section{REFERENCES}

1. Kisner C, Colby LA, Borstad J. Therapeutic exercise: foundations and techniques. 7th ed. Philadelphia: F.A. Davis; 2018.

2. Ludewig PM, Cook TM. Alterations in shoulder kinematics and associated muscle activity in people with symptoms of shoulder impingement. Phys Ther 2000;80(3):276-91.

3. McClure PW, Bialker J, Neff N, Williams G, Karduna A. Shoulder function and 3-dimensional kinematics in people with shoulder impingement syndrome before and after a 6-week exercise program. Phys Ther 2004;84(9):832-48.

4. McClure PW, Michener LA, Karduna AR. Shoulder function and 3-dimensional scapular kinematics in people with and without shoulder impingement syndrome. Phys Ther 2006;86(8):1075-90.

5. Ludewig PM, Cook TM, Nawoczenski DA. Three-dimensional scapular orientation and muscle activity at selected positions of humeral elevation. J Orthop Sports Phys Ther 1996;24(2):57-65.

6. Decker MJ, Hintermeister RA, Faber KJ, Hawkins RJ. Serratus anterior muscle activity during selected rehabilitation exercises. Am J Sports Med 1999;27(6):784-91.

7. Inman VT, Saunders JB, Abbott LC. Observations of the function of the shoulder joint. Clin Orthop Relat Res 1996;(330):312.

8. Wiater JM, Flatow EL. Long thoracic nerve injury. Clin Orthop Relat Res 1999;(368):17-27.

9. Warner JJ, Navarro RA. Serratus anterior dysfunction. Recognition and treatment. Clin Orthop Relat Res 1998;(349):13948.

10. Kibler WB. The role of the scapula in athletic shoulder function. Am J Sports Med 1998;26(2):325-37.

11. Magee DJ, Manske RC. Orthopedic physical assessment. 7th ed. St. Louis: Elsevier; 2021.

12. Falsone SA, Gross MT, Guskiewicz KM, Schneider RA. One-arm hop test: reliability and effects of arm dominance. J Orthop Sports Phys Ther 2002;32(3):98-103.

13. Gorman PP, Butler RJ, Plisky PJ, Kiesel KB. Upper quarter Y balance test: reliability and performance comparison between genders in active adults. J Strength Cond Res 2012; 26(11):3043-8.

14. Tucci HT, Martins J, de Carvalho Sposito G, Camarini PM, de Oliveira AS. Closed kinetic chain upper extremity stability test (CKCUES test): a reliability study in persons with and without shoulder impingement syndrome. BMC Musculoskelet Disord 2014;15:1

15. Roush JR, Kitamura J, Waits MC. Reference values for the closed kinetic chain upper extremity stability test (CKCUEST) for collegiate baseball players. N Am J Sports Phys Ther 2007;2(3):159-63.

16. Dennis RJ, Finch CF, Elliott BC, Farhart PJ. The reliability of musculoskeletal screening tests used in cricket. Phys Ther Sport 2008;9(1):25-33

17. Plisky PJ, Gorman PP, Butler RJ, Kiesel KB, Underwood FB, Elkins B. The reliability of an instrumented device for measuring components of the star excursion balance test. N Am J Sports Phys Ther 2009;4(2):92-9. 
18. de Oliveira VM, Pitangui AC, Nascimento VY, da Silva HA, Dos Passos MH, de Araújo RC. Test-retest reliability of the closed kinetic chain upper extremity stability test (CKCUEST) in adolescents: reliability of ckcuest in adolescents. Int J Sports Phys Ther 2017;12(1):125-32.

19. Goldbeck TG, Davies GJ. Test-retest reliability of the closed kinetic chain upper extremity stability test: a clinical field test. J Sport Rehabil 2000;9(1):35-45.

20. Lee DR, Kim LJ. Reliability and validity of the closed kinetic chain upper extremity stability test. J Phys Ther Sci 2015; 27(4):1071-3.

21. Kim HA, Hwang UJ, Jung SH, Ahn SH, Kim JH, Kwon OY. Comparison of shoulder strength in males with and without myofascial trigger points in the upper trapezius. Clin Biomech (Bristol, Avon) 2017;49:134-8.

22. Francis P, Gray K, Perrem N. The relationship between concentric hip abductor strength and performance of the Y-balance test (YBT). Int J Athl Ther Train 2018;23(1):42-7.

23. Park SY, Yoo WG. Differential activation of parts of the serratus anterior muscle during push-up variations on stable and unstable bases of support. J Electromyogr Kinesiol 2011; 21(5):861-7.

24. Pirauá AL, Pitangui AC, Silva JP, Pereira dos Passos MH, Alves de Oliveira VM, Batista Lda S, et al. Electromyographic analysis of the serratus anterior and trapezius muscles during push-ups on stable and unstable bases in subjects with scapular dyskinesis. J Electromyogr Kinesiol 2014;24(5):675-81.

25. Ludewig PM, Hoff MS, Osowski EE, Meschke SA, Rundquist PJ. Relative balance of serratus anterior and upper trapezius muscle activity during push-up exercises. Am J Sports Med
2004;32(2):484-93.

26. Moseley JB Jr, Jobe FW, Pink M, Perry J, Tibone J. EMG analysis of the scapular muscles during a shoulder rehabilitation program. Am J Sports Med 1992;20(2):128-34.

27. Park KM, Cynn HS, Kwon OY, Yi CH, Yoon TL, Lee JH. Comparison of pectoralis major and serratus anterior muscle activities during different push-up plus exercises in subjects with and without scapular winging. J Strength Cond Res 2014;28(9): 2546-51.

28. Escamilla RF, Yamashiro K, Paulos L, Andrews JR. Shoulder muscle activity and function in common shoulder rehabilitation exercises. Sports Med 2009;39(8):663-85.

29. Celik D, Sirmen B, Demirhan M. The relationship of muscle strength and pain in subacromial impingement syndrome. Acta Orthop Traumatol Turc 2011;45(2):79-84.

30. Taylor JB, Wright AA, Smoliga JM, DePew JT, Hegedus EJ. Upper-extremity physical-performance tests in college athletes. J Sport Rehabil 2016;25(2):146-54.

31. Degot M, Blache Y, Vigne G, Juré D, Borel F, Neyton L, et al. Intrarater reliability and agreement of a modified Closed Kinetic Chain Upper Extremity Stability Test. Phys Ther Sport 2019;38:44-8.

32. Torres RJB, Pirauá ALT, Nascimento VYS, Dos Santos PS, Beltrão NB, de Oliveira VMA, et al. Shoulder muscle activation levels during the push-up-plus exercise on stable and unstable surfaces. J Sport Rehabil 2017;26(4):281-6.

33. Lehman GJ, MacMillan B, MacIntyre I, Chivers M, Fluter M. Shoulder muscle EMG activity during push up variations on and off a Swiss ball. Dyn Med 2006;5:7. 